\title{
Resonant coupling of mode-1 and mode-2 internal waves by topography
}

\author{
Zihua Liu $^{1} \dagger$, Roger Grimshaw ${ }^{1}$ and Edward Johnson ${ }^{1}$ \\ ${ }^{1}$ Department of Mathematics, University College London, London, WC1E 6BT, UK.
}

(Received $\mathrm{xx}$; revised $\mathrm{xx}$; accepted $\mathrm{xx}$ )

We consider the resonant coupling of mode- 1 and mode- 2 internal solitary waves by topography. Mode- 2 waves are generated by a mode- 1 wave encountering variable topography, modelled by coupled Korteweg-de Vries $(\mathrm{KdV})$ system. Three cases $(A)$ weak resonant coupling, $(B)$ moderate resonant coupling, $(C)$ strong resonant coupling, are examined in detail using a three-layer density-stratified fluid system with different stratification and topographic settings. The strength of the resonant coupling is determined by the range of values taken by the ratio of linear long wave phase speeds $\left(c_{2} / c_{1}\right.$ where $c_{1}$ is the mode- 1 speed and $c_{2}$ the mode- 2 speed) while the waves are above the slope. In case $(A)$ the range is from 0.42 (ocean edge) to 0.48 (shelf edge), in case $(B)$ from 0.58 (ocean) to 0.72 (shelf), and in case $(C)$ from 0.44 (ocean) to 0.92 (shelf). The feedback from mode- 2 to mode- 1 is estimated by comparing coupled KdV system with a $\mathrm{KdV}$ model. In case $(A)$, a small-amplitude convex mode- 2 wave is generated by a depression mode- 1 wave and the feedback on the mode- 1 wave is negligible. In case $(B)$, a concave mode- 2 wave of comparable amplitude to the depression incident mode- 1 wave is formed; strong feedback enhances the polarity change process of the mode- 1 wave. In case $(C)$, a large-amplitude concave mode- 2 wave is produced by an elevation mode- 1 wave; strong feedback suppresses the fission of the mode-1 wave. Simulations for a wider range of topographic slopes and three-layer stratifications are then classified in terms of these responses.

Key words:

\section{Introduction}

Internal waves that propagate along sharp density gradients (analogous to surface waves where the density gradient is from water to air) are commonly observed in the coastal ocean, lakes and fjords. Theoretically, horizontally propagating internal waves can be decomposed into an infinite set of vertical modes whose linear long wave phase speeds decrease with an increase in the mode number, as the vertical structure increases in complexity. Most studies have focussed on mode- 1 internal solitary waves as they are the most frequently observed in the ocean (Liu et al. (1998); Klymak and Moum (2003); Moum and Smyth (2006) and Zhao and Alford (2006)). However, recently there has been more interest in mode-2 internal solitary waves with several observations (Farmer and Smith (1980); Yang et al. (2009, 2010); Shroyer et al. (2010); Liu et al. (2013); Ramp et al. (2012); Magalhaes and Da Silva (2018) and Rayson et al. (2019)). Mode-1 and mode-2 waves are defined by their linear long wave phase speeds $c_{1}, c_{2}$ respectively with $c_{2}<c_{1}$.

$\dagger$ Email address for correspondence: zihua.liu.15@ucl.ac.uk 
They are also distinguished from their shapes, as mode-1 waves displace isopycnals in one direction only, while mode- 2 waves displace the upper and lower isopycnals in opposite directions, which can be convex or concave. In general, as lower mode waves travel faster than higher mode waves, from a localised initial condition the modes spread out and each mode develops into solitary waves. However, in some special circumstances when $c_{2} \approx c_{1}$ (although always $c_{2}<c_{1}$ ), the mode- 2 waves will interact resonantly with mode- 1 waves over a long time scale. This scenario is the topic of this paper.

As for the many studies of mode- 1 internal solitary waves, generation mechanisms and the evolution of mode-2 internal solitary waves over topography are now receiving more attention. Helfrich and Melville (1986) found that the instability of a shoaling mode-1 wave may lead to the generation of a mode-2 wave. Vlasenko and Hutter (2001) demonstrated experimentally and theoretically that a reflected and transmitted mode- 2 wave may emerge when a mode- 1 wave interacts with a localised sill. A detailed study of shoaling internal solitary waves under different background environments in the South China Sea was reported by Lamb and Warn-Varnas (2015), showing that a mode-2 wave could be generated at small-scale features in the bathymetry. Recently we examined the interaction of a mode-1 internal solitary wave with slope-shelf topography generating mode-2 waves, with an amplitude sensitive to the stratification layer thicknesses and topographic slope, Liu et al. (2019a). Another mechanism for the generation of mode-2 waves is local generation, that is the release of a density front into a pycnocline which is often used in laboratory and numerical studies (Maxworthy (1980); Mehta et al. (2002); Deepwell and Stastna (2016); Rayson et al. (2019) and Carr et al. (2019)). There are several studies of the transformation of a mode-2 solitary wave over bottom topography, for example, a step (Terletska et al. (2016), Liu et al. (2019b)), a narrow ridge (Deepwell et al. (2017)), a broad ridge Deepwell et al. (2019), a uniform slope (Carr et al. (2019)) and a slope-shelf (Cheng et al. (2017); Yuan et al. (2018)).

Mode-2 waves will interact with other wave modes, such as mode-1 waves, through nonlinearity in the underlying fluid system. Propagating mode- 2 solitary waves with an accompanying tail of mode- 1 waves have been observed in field experiments (Farmer and Smith (1980) and Shroyer et al. (2010)), and examined theoretically by Akylas and Grimshaw (1992) and numerically by Vanden-Broeck and Turner (1992). Gear and Grimshaw (1984) studied the interaction between internal solitary wave modes using coupled $\mathrm{KdV}$ equations, finding that weak or strong interactions happen when the wave phase speeds are unequal or nearly equal, respectively. A numerical investigation was presented by Stastna et al. (2015) on the strong mode-mode coupling between mode-1 and mode-2 internal solitary waves with both head-on and overtaking collisions, showing that the mode- 2 wave structure can be significantly deformed by the mode- 1 wave during the collision. Maderich et al. (2017) investigated the head-on collision of three types of internal solitary waves with trapped cores numerically based on the framework of the Navier-Stokes equation for a stratified fluid.

In this paper, we consider the generation of mode- 2 waves as a mode- 1 wave propagates shoreward from deep water, over the continental slope into shallow water, and in particular the resonant coupling by topography of the generated mode- 2 wave and the incident mode-1 wave. In (Liu et al. (2019a)) we adapted the linear long wave theory developed by Griffiths and Grimshaw (2007) which describes the topographic coupling of a full set of linear long-wave modes. We took an incident mode-1 solitary wave which then generated a mode-2 wave when it encountered the variable topography of the continental slope. Once the mode-2 wave is formed, we used a Korteweg-de Vries (KdV) equation (2.1) to follow its evolution over the continental slope and shelf. This approach is valid when the linear phase speeds are quite distinct. Here we extend that study to the case when 
instead the mode- 1 and mode- 2 linear phase speeds are close, that is, $c_{2} \approx c_{1}$, noting that always $c_{2}<c_{1}$. This gives resonant coupling and leads to coupled KdV equations, incorporating feedback from the generated mode- 2 wave on the incident mode- 1 wave.

In $\S 2$, we introduce the theoretical methods, the $\mathrm{KdV}$ equation for internal solitary waves, and coupled modes in both a linearised system and in a system of coupled KdV equations to describe the generation of mode- 2 internal solitary waves and the resonant coupling with the incident mode- 1 wave by slope-shelf topography. We have introduced the noted $\mathrm{KdV}$ equation and linear long wave theory with mode coupling in Liu et al. (2019a), that the coupled KdV system is based on. For convenience we show the details in this paper again using the same notations and expressions. In $\S 3$, we apply this theory to a three-layer density-stratified fluid system, for different layer thicknesses and topographic slopes that determine the mode- 1 and mode- 2 linear phase speeds, and hence the range over the slope of the ratio of the speed $c_{2} / c_{1}$, which is one of the key parameters in the coupling term. In general, since mode- 2 waves are slower in speed, typically onethird of the speed of mode- 1 wave, we vary the setup of background stratification and/or topographic gradient to increase the range of the ratio of $c_{2} / c_{1}$ and $\frac{\partial h}{\partial T}$ and hence get strong resonant coupling. Three examples $(A, B$ and $C$ in $\S 3)$ are considered in detail with the speeds $c_{2}$ and $c_{1}$ becoming closer, while in each case two different topographic slopes are examined, gentle and steep. Simulations over wider ranges of stratification and topography are then summarised in terms of these three cases. $\S 4$ discusses the results and we summarise in $\S 5$.

\section{Theoretical formulation}

\subsection{Korteweg-de Vries equation}

The usual KdV equation for propagation of weakly nonlinear long internal waves over variable topography with depth $h(x)$ is, in standard notation,

$$
\frac{\partial A}{\partial t}+c \frac{\partial A}{\partial x}+\frac{1}{2 Q} \frac{\partial Q}{\partial x} c A+\mu A \frac{\partial A}{\partial x}+\delta \frac{\partial^{3} A}{\partial x^{3}}=0,
$$

see Grimshaw (1981), and the reviews by Helfrich and Melville (2006), Grimshaw (2007) and Grimshaw et al. (2010). Here $\zeta=A(x, t) \phi(z ; h)$ is the leading order expression for the vertical particle displacement. The modal function $\phi(z ; h)$ is determined by, using the Boussinesq approximation, a rigid upper lid approximation, and in the absence of a background shear flow,

$$
\frac{\partial^{2} \phi}{\partial z^{2}}+\frac{N^{2}}{c^{2}} \phi=0 ; \quad \phi=0, \quad z=0,-h(x) .
$$

Here $N^{2}=-\frac{g}{\rho_{0}} \frac{\mathrm{d} \rho_{0}}{\mathrm{~d} z}$ is the background density field. This modal equation determines the modal function $\phi(z ; h)$ and the linear long wave speed $c(h)$ where the $h$-dependence is parametric, since it is assumed that the depth $h(x)$ is slowly varying. The coefficients in (2.1) are given by, in the same approximation used in (2.2),

$$
\begin{gathered}
I \mu=3 c^{2} \int_{-h}^{0} \rho_{0}\left(\frac{\partial \phi}{\partial z}\right)^{3} d z \\
I \delta=c^{2} \int_{-h}^{0} \rho_{0} \phi^{2} d z \\
I=2 c \int_{-h}^{0} \rho_{0}\left(\frac{\partial \phi}{\partial z}\right)^{2} d z, \quad Q=c^{2} I .
\end{gathered}
$$

In general, the modal equation (2.2) determines an infinite set of modes $\phi_{n}, n=$ $1,2, \cdots$, ordered so that $c_{1}>c_{2}>\cdots$. Usually only mode- $1, \phi_{1}$ with the fastest linear 
phase speed $c_{1}$ is considered. Then the KdV equation (2.1) describes the evolution of the amplitude of this mode. But importantly we note that the same KdV equation (2.1) can be used for a mode- 2 wave, with coefficients determined by $(2.3,2.4,2.5)$ using the mode- 2 modal function $\phi_{2}$ and speed $c_{2}<c_{1}$.

\subsection{Coupled modes: Linearised system}

The theory of Griffiths and Grimshaw (2007) uses linear long wave theory to decompose the wave field into a sum of vertical modes, noting that the full set of modal functions defined by (2.2) are complete. This was used by Liu et al. (2019a) to examine the generation of a mode- 2 wave by a mode- 1 wave incident on the topography. As in Liu et al. (2019a) the theory of Griffiths and Grimshaw (2007) is restricted to just two modes, so that the wave field is given by

$$
\begin{aligned}
& \zeta=\sum_{n=1}^{n=2} A_{n}(x, t) \phi_{n}(z ; h), \\
& u=\sum_{n=1}^{n=2} A_{n}(x, t) c_{n}(h) \frac{\partial \phi_{n}(z ; h)}{\partial z}, \\
& p=\rho_{0} \sum_{n=1}^{n=2} A_{n}(x, t) c_{n}^{2}(h) \frac{\partial \phi_{n}(z ; h)}{\partial z} .
\end{aligned}
$$

Here $\zeta, u, p$ are the vertical particle displacement, the horizontal velocity and the dynamic pressure respectively. The vertical velocity is $w=\frac{\partial \zeta}{\partial t}$ and the density perturbation is $\rho=N^{2} \zeta / g$. From Griffiths and Grimshaw (2007) (see equation (24)) the linear long wave coupling between modes- 1 and mode- 2 with linear long wave speeds $c_{1,2}$ is given by, for slowly-varying $h(x)$,

$$
\begin{gathered}
\frac{1}{c_{1}^{2}} \frac{\partial^{2} U_{1}}{\partial t^{2}}-\frac{\partial^{2} U_{1}}{\partial x^{2}}=-\gamma \frac{\partial h}{\partial x} \frac{\partial U_{2}}{\partial x} \\
\frac{1}{c_{2}^{2}} \frac{\partial^{2} U_{2}}{\partial t^{2}}-\frac{\partial^{2} U_{2}}{\partial x^{2}}=\gamma \frac{\partial h}{\partial x} \frac{\partial U_{1}}{\partial x}, \\
\gamma=\frac{2}{\tilde{\lambda}_{1}-\tilde{\lambda}_{2}}\left(\frac{\partial \tilde{\lambda}_{1}}{\partial h} \frac{\partial \tilde{\lambda}_{2}}{\partial h}\right)^{1 / 2}=\frac{4}{c_{2}^{2}-c_{1}^{2}}\left(c_{1} c_{2} \frac{\partial c_{1}}{\partial h} \frac{\partial c_{2}}{\partial h}\right)^{1 / 2}, \quad \tilde{\lambda}_{1}=\frac{1}{c_{1}^{2}}, \quad \tilde{\lambda}_{2}=\frac{1}{c_{2}^{2}} .
\end{gathered}
$$

As noted above, this is a reduction from the full set in equation (24) of Griffiths and Grimshaw (2007) by restriction to just two modes, and retention of only the leading order topographic coupling terms when $h(x)$ is slowly varying; that is, terms such as $\frac{\partial^{2} h}{\partial x^{2}}$ or $\left(\frac{\partial h}{\partial x}\right)^{2}$ are omitted. However, the theory of Griffiths and Grimshaw (2007) was developed for the horizontal velocity field in the form $u=U \frac{\partial \psi(z ; h)}{\partial z}$, whereas in the usual $\mathrm{KdV}$ theory described above by (2.6) the theory is developed for the vertical particle displacement $A \phi(z ; h)$, where the modal function $\psi \propto \phi$. In the absence of a background shear flow, these are related by, temporarily omitting the modal index,

$$
u=U \frac{\partial \psi}{\partial z}=c A \frac{\partial \phi}{\partial z}, \quad \psi(z)=K \phi(z), \quad K^{2}=\frac{1}{I c}=\frac{c}{Q} .
$$

Hence we find that

$$
U=c^{1 / 2} B, \quad B=Q^{1 / 2} A,
$$


where $Q, I$ are the linear magnification and normalisation factors in the usual KdV theory, see $(2.5)$.

Since $h(x)$ is slowly varying, we use the change of variables,

$$
\begin{gathered}
T=\int_{0}^{x} \frac{d x}{c_{1}}, \quad X=T-t . \\
\frac{\partial U}{\partial x}=\frac{1}{c_{1}}\left(\frac{\partial U}{\partial X}+\frac{\partial U}{\partial T}\right), \quad \frac{\partial^{2} U}{\partial t^{2}}=\frac{\partial^{2} U}{\partial X^{2}} \\
\frac{\partial^{2} U}{\partial x^{2}}=\frac{1}{c_{1}^{2}}\left(\frac{\partial^{2} U}{\partial X^{2}}+2 \frac{\partial^{2} U}{\partial X \partial T}+\frac{\partial^{2} U}{\partial T^{2}}\right)-\frac{1}{c_{1}^{3}} \frac{\partial c_{1}}{\partial T}\left(\frac{\partial U}{\partial X}+\frac{\partial U}{\partial T}\right) .
\end{gathered}
$$

In Liu et al. (2019a) we used (2.12) for mode-1 and an analogous change of variable for mode-2 with $c_{1}$ replaced with $c_{2}$. Here we assume that $c_{1} \approx c_{2}$, and so we can use (2.12) for both modes. Then in each modal equation, the operator on the left-hand side becomes

$$
\frac{1}{c_{1}^{2}} \frac{\partial^{2} U}{\partial t^{2}}-\frac{\partial^{2} U}{\partial x^{2}}=-\frac{2}{c_{1}^{2}} \frac{\partial^{2} U}{\partial X \partial T}-\frac{1}{c_{1}^{2}} \frac{\partial^{2} U}{\partial T^{2}}+\frac{1}{c_{1}^{3}} \frac{\partial c_{1}}{\partial T}\left(\frac{\partial U}{\partial X}+\frac{\partial U}{\partial T}\right) .
$$

Next we use the transformation (2.11) and assume that the $T$-derivatives vary slowly relative to $X$-derivatives. Thus we can neglect slowly-varying terms such as $\frac{\partial^{2} U}{\partial T^{2}}, \frac{\partial c_{1}}{\partial T} \frac{\partial U}{\partial T}$, and the coupled system $(2.7,2.8)$ becomes

$$
\begin{gathered}
\frac{\partial B_{1}}{\partial T}=\frac{\gamma c_{2}^{1 / 2}}{2 c_{1}^{1 / 2}} \frac{\partial h}{\partial T} B_{2} \\
\frac{\partial B_{2}}{\partial T}-\Delta \frac{\partial B_{2}}{\partial X}=-\frac{\gamma c_{1}^{1 / 2}}{2 c_{2}^{1 / 2}} \frac{\partial h}{\partial T} B_{1}, \quad \Delta=\left\{\frac{1}{c_{2}^{2}}-\frac{1}{c_{1}^{2}}\right\} \frac{c_{1}^{2}}{2} \approx\left(c_{1}-c_{2}\right) / c_{2} .
\end{gathered}
$$

In the original $x, t$ variable there is a choice of either an initial condition, when $B_{1}, B_{2}$ are specified at $t=0$, or a boundary condition when $B_{1}, B_{2}$ are at $x=0$. Here, we choose $B_{1}$ to be a mode- 1 solitary wave, either located in $x<0$ at $t=0$ or specified at $x=0$ and in both cases $B_{2}=0$. In the initial-value problem, these initial conditions are located at $X=T$, or as boundary conditions at $T=0$. These can be shown to be asymptotically equivalent with respect to the small long-wave parameter used to derive $(2.7,2.8)$, and also the $\mathrm{KdV}$ equation $(2.1)$, see below in the next subsection, But, nevertheless they produce different solutions in general. Here we choose the boundary condition formulation, inter alia being more convenient for numerical simulations. Thus the "initial" condition at $T=0$ is a mode- 1 solitary wave, defined in $T<0$ by $B_{1}=$ $B_{1 \text { sol }}(X-V T)$ in $T<0$ where $h=h_{b}$, and a zero mode- 2 wave, $B_{2}=0$. Here $V$ is the solitary wave speed (see below (2.25)), and in the linear long wave limit we set $V=0$. Importantly, note that $\Delta>0$ since $c_{1}>c_{2}$, and we have used the approximation that $c_{2} \approx c_{1}$ to simplify $\Delta$.

\subsection{The linear response}

The coupled system $(2.15,2.16)$ forms the bases of the investigation here. An estimate of the size of a typical solution of this system can be found using the method in Liu et al. (2019a) to solve the linear system approximately when $\Delta$ is of order unity. Since the coupling term $\gamma \frac{\partial h}{\partial T}$ is slowly varying and hence small, it can be neglected in (2.15) when the incident wave is a mode- 1 wave. It then follows that to leading order $B_{1}=B_{1 \text { sol }}(X)$ is independent of $T$ and determined from the initial condition of a mode- 1 solitary wave 
a priori. The second equation (2.16) can then be solved for the mode- 2 wave,

$$
\begin{aligned}
& B_{2} \approx-\int_{0}^{\tau} C\left(\tau^{\prime}\right) B_{1 s o l}\left(X+\tau-\tau^{\prime}\right) d \tau^{\prime} \\
& \tau(T)=\int_{0}^{T} \Delta\left(T^{\prime}\right) d T^{\prime}, \quad C(\tau)=\frac{\gamma c_{1}^{1 / 2}}{2 c_{2}^{1 / 2} \Delta} \frac{\partial h}{\partial T} .
\end{aligned}
$$

Note that the evolution variable $T$ is replaced with $\tau$ and the "initial" condition that $B_{2}=0, T=0$, that is $B_{2}=0, \tau=0$ has been imposed. This can be written in the alternative form, putting $Y=X+\tau-\tau^{\prime}$, so that $X<Y<X+\tau$ when $\tau>\tau^{\prime}>0$,

$$
B_{2} \approx-\int_{X}^{X+\tau} C(X+\tau-Y) B_{1 s o l}(Y) d Y .
$$

This form indicates that this approximate solution is a combination of a slaved mode-2 wave depending on $X$ and a free mode-2 wave depending on $X+\tau$. Note that because $C(\tau) \geqslant 0, B_{2}$ has the opposite sign to $B_{1 s o l}$.

Since $C$ is slowly varying compared to $B_{1 \text { sol }}, B_{1 \text { sol }}(X)$ can be regarded as localised around $X=0$, and then the expression (2.18) for $B_{2}$ is effectively confined to the region $-\tau<X<0$, where the two boundaries correspond to a free wave and a slaved wave respectively. For instance if we approximate $B_{1}(X)$ with a $\delta$-function, $B_{1}(X)=a \delta(K X)$ with mass $a / K$, then $(2.18)$ becomes

$$
B_{2} \approx-\frac{a}{K} C(X+\tau)\{\mathcal{H}(X+\tau)-\mathcal{H}(X)\}
$$

where $\mathcal{H}(\cdot)$ is the Heaviside function. For each fixed $\tau>0$, as $X$ increases over the range $-\tau<X<0, X+\tau$ increases from 0 to $\tau$. and this approximate solution (2.19) is non-zero only for $-\tau<X<0$. The maximum amplitude of $B_{2}$ is $C_{M} a / K$ where $C_{M}=\max [C]$. Further from (2.17), $C(\tau(T))$ is non-zero only on the slope, that is, $0<\tau(T)<\tau(L)$. Hence (2.19) is non-zero only for $-\tau(T)<X<0$, and for $-\tau(T)<X<\tau(T)-\tau(L)$. In these combined regions, (2.19) is a function of $X+\tau$ and so is a free wave. On the other hand, if $C$ is rapidly varying relative to $B$, as would be the case if $h(T)$ is close to being a step, then we might approximate $C$ with a $\delta$-function $C(\tau)=C_{M} \delta\left(L\left(\tau-\tau_{0}\right)\right)$. Then the solution (2.17) becomes

$$
B_{2} \approx-\frac{C_{M}}{L} B_{1 s o l}\left(X+\tau-\tau_{0}\right) .
$$

This is a free wave and importantly note that compared to (2.19) the amplitude varies as $a C_{M} / L$ rather than $a C_{M} / K$.

These approximate solutions of $(2.15,2.16)$ can be compared with those presented in Liu et al. (2019a) where it was assumed that $\Delta$ is order unity, and the $T$-derivative in (2.16) was omitted on the basis that the forcing term $B_{1 \text { sol }}(X)$ depended only on $X$. Also, crucially, in Liu et al. (2019a) the initial condition that $B_{2}=0$ was imposed at $t=0, X=T$ instead of at $T=0$. As already noted, although these initial conditions are asymptotically equivalent, they lead in practice to different outcomes. In the present notation the solution found by Liu et al. (2019a) is

$$
\begin{aligned}
& B_{1} \approx B_{1 s o l}(X), \quad B_{2} \approx B_{2 P}(X, \tau)+B_{2 F}(X+\tau), \\
& B_{2 P}=-C(\tau) \int_{X}^{\infty} B_{1 s o l}\left(X^{\prime}\right) d X^{\prime}, \\
& B_{2 F}(Z)=-\begin{array}{l}
B_{2 P}(X, \tau(X)), \quad Z(X)=X+\tau(X) . \\
\text { Cambridge University Press }
\end{array}
\end{aligned}
$$




\begin{tabular}{|c|c|c|c|c|c|c|}
\hline Case & $h_{1}$ & $h_{2}$ & $\begin{array}{c}h_{3} \\
\text { off-shelf }\end{array}$ & $\begin{array}{c}h_{3} \\
\text { on-shelf }\end{array}$ & $\begin{array}{c}h_{\text {gentle }} \\
\text { slope width }\end{array}$ & $\begin{array}{c}h_{\text {steep }} \\
\text { slope width }\end{array}$ \\
\hline$A$ & 120 & 80 & 300 & 150 & 8000 & 2000 \\
\hline$B$ & 84 & 180 & 236 & 86 & 8000 & 2000 \\
\hline$C$ & 24 & 300 & 176 & 26 & 8000 & 2000 \\
\hline
\end{tabular}

TABLE 1. The layer depths (in metres) of the three-layer flow for the depth profiles $h_{\text {gentle }}$ and $h_{\text {steep }}$ of (3.1) and (3.2). The slope width is defined as the position where the argument of tanh in $(3.1)$ and (3.2) is 4 .

(a)

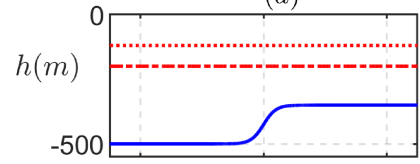

$(d)$

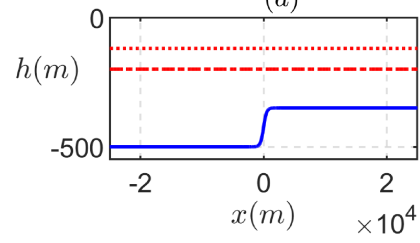

(b)

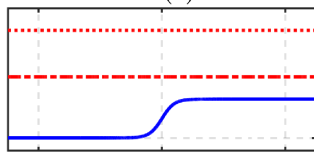

$(e)$

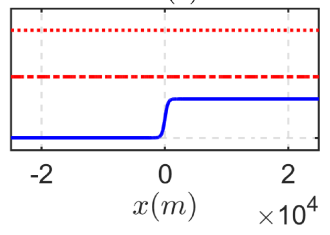

$(c)$

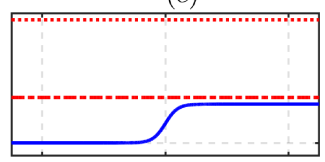

$(f)$

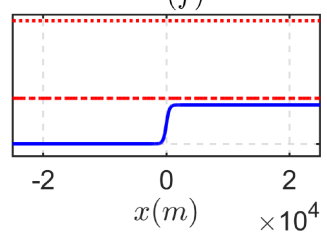

Figure 1. Setup of the three-layer fluid model with depth profiles given by (3.1, 3.2). Panels $(a, b, c)$ and $(d, e, f)$ are for gentle and steep slopes, respectively. For layer thicknesses, panel $(a, d): A$; panels $(b, e): B$; panels $(c, f): C$.

Here in the expression $\tau=\tau(T)$ in (2.17), we put $X=T$, and then an inversion is needed to get $X=X(Z)$, so that the functional form of $B_{2 F}(Z)$ can be determined. Although there is an obvious similarity between (2.18) and (2.21) they are not the same. However, the underlying assumption here is that the $T$-scale is slowly varying compared to the $X$-scale, and in that limit the two expressions agree. Indeed, if $C(\tau)$ is taken to be a constant, then they are identical, indicating that if $C(\tau)$ is sufficiently slowly varying, then the two expression are close. The linear response of the present solution when $\Delta$ is not small thus merges smoothly with the linear results of Liu et al. (2019a).

\subsection{Coupled modes: Korteweg-de Vries system}

The present study concerns the behaviour when $c_{2} \approx c_{1}$ so that $\Delta \ll 1$, and also then $\gamma \propto \Delta^{-1}$ becomes large, see (2.9). In this limit the coupled system $(2.15,2.16)$ yields high frequency oscillations with a frequency proportional to $\Delta^{-1}$ and a wavenumber proportional to $\Delta^{-2}$. This resonance leads to the requirement that weakly nonlinear terms need to be invoked, together with weak linear dispersion, requiring the $\mathrm{KdV}$ extension of the coupled system, examined here.

In the absence of topographic coupling we can add a $\mathrm{KdV}$ extension for each mode, 
that is

$$
\frac{\partial B}{\partial T} \rightarrow \frac{\partial B}{\partial T}+\nu B \frac{\partial B}{\partial X}+\lambda \frac{\partial^{3} B}{\partial X^{3}}, \quad \nu=\frac{\mu}{c Q^{1 / 2}} \quad \lambda=\frac{\delta}{c^{3}},
$$

after applying the transformations (2.12) to the $\mathrm{KdV}$ equation, and (2.1), and $\mu, \lambda$ are defined by $(2.3,2.4)$ for each mode. Hence we propose that the coupled KdV system for this resonance will be

$$
\begin{gathered}
\frac{\partial B_{1}}{\partial T}+\nu_{1} B_{1} \frac{\partial B_{1}}{\partial X}+\lambda_{1} \frac{\partial^{3} B_{1}}{\partial X^{3}}=\frac{\gamma c_{2}^{1 / 2}}{2 c_{1}^{1 / 2}} \frac{\partial h}{\partial T} B_{2} \\
\frac{\partial B_{2}}{\partial T}-\Delta \frac{\partial B_{2}}{\partial X}+\nu_{2} B_{2} \frac{\partial B_{2}}{\partial X}+\lambda_{2} \frac{\partial^{3} B_{2}}{\partial X^{3}}=-\frac{\gamma c_{1}^{1 / 2}}{2 c_{2}^{1 / 2}} \frac{\partial h}{\partial T} B_{1} .
\end{gathered}
$$

The usual KdV scaling requires that $B_{1,2} \sim \epsilon^{2}, \partial / \partial X \sim \epsilon$ and $\partial / \partial T \sim \epsilon^{3}$. Here in addition we require a resonance so that $\Delta \sim \epsilon^{2}$ and so then $\gamma \frac{\partial h}{\partial T} \sim \epsilon^{3}$. From (2.9) $\gamma \propto \Delta^{-1}$, it follows that formally $\frac{\partial h}{\partial T} \sim \epsilon^{5}$. Note that although we are assuming that $c_{2} \approx c_{1}$, the modal functions $\phi_{1}$ and $\phi_{2}$ remain distinct. It is consistent asymptotically to replace $\Delta$ in $(2.16)$ with $\Delta \approx\left(c_{1}-c_{2}\right) / c_{2}$, and also put $c_{2} / c_{1} \approx 1$ in the coupling terms on the right-hand side. Also it is useful to note that in this $X-T$ reference frame, the linear phase speeds for the mode- 1 and mode- 2 waves are $0,-\Delta$ respectively.

The "initial" condition at $T=0$ is that the mode- 2 is zero, $B_{2}=0$, and there is only a mode- 1 solitary wave in $T \leqslant 0$, that is,

$$
B_{1}=a \operatorname{sech}^{2}(K(X-V T)), \quad V=\frac{a \nu_{1 b}}{3}=4 K^{2} \lambda_{1 b}, \quad B_{2}=0 .
$$

\section{Typical behaviours}

We consider a density-stratified three-layer fluid model as in Liu et al. (2019a), where we found the generated mode- 2 wave amplitude is quite sensitive to the pycnocline thickness and the topographic slope. With this same three-layer fluid model, we choose a suitable expressions for the depth profile $h(x)$, and the layer depths $h_{1}$ and $h_{2}$. Two depth profiles are set with distinct topographic gradients:

$$
h(x)_{\text {gentle }}=425-75 \tanh \left(5 \cdot 10^{-4} x\right) \quad(m),
$$

and

$$
h(x)_{\text {steep }}=425-75 \tanh \left(2 \cdot 10^{-3} x\right) \quad(m) .
$$

Each represents a smooth transition from a deep water depth of $500 \mathrm{~m}$ to a shallow water depth of $350 \mathrm{~m}$, with either a gentle (3.1) or a steep (3.2) slope, respectively. Note that the origin of $x$ is at mid-slope here. We have found that the combination of a near-surface and a near-bottom pycnocline is a scenario favouring the generation of mode- 2 waves, see Liu et al. (2019a). Three cases of the top and middle layer thicknesses are chosen for the each of the gentle (3.1) and steep (3.2) slope cases, see table 2.4. The densities in each layer are $\rho_{0}-\Delta \rho, \rho_{0}, \rho_{0}+\Delta \rho$ respectively. The density stratifications are chosen to be close to the common oceanic conditions and to capture the complete range of responses expected from an incident mode-1 wave.

Figure 1 shows the set up of this three-layer fluid model with different depth profiles and pycnocline thicknesses. For cases $A$ and $B$, initially there is a mode- 1 depression solitary wave, with a polarity change for case $B$, while for case $C$, initially there is a mode-1 elevation solitary wave. In the following subsections we present the wave evolution 
(a)
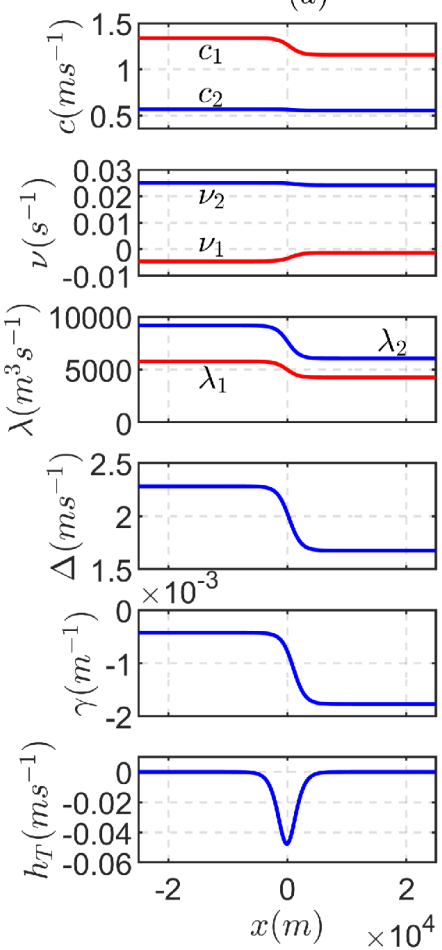

(b)
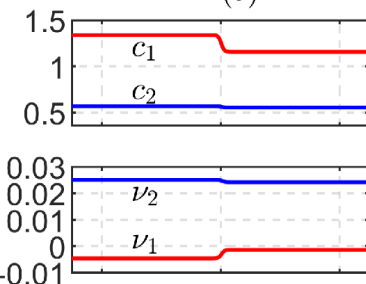

$-0.01$
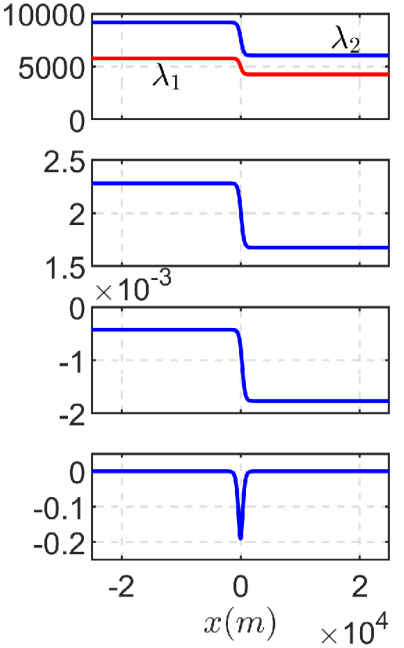

Figure 2. Case $A$. Coefficients of the coupled-KdV $(2.23,2.24)$ model. Panels $(a, b)$ correspond to the gentle and steep slope respectively.

results for both the coupled-KdV system $(\S 2.4)$ and for comparison the KdV evolution for the mode-1 wave alone with no topographic coupling (§2.1), in each of these six different situations. The magnitude of the initial mode- 1 solitary wave amplitude is $5 \mathrm{~m}$, either depression or elevation. Resonant coupling is expected to be significant when the range over the topography of the speed ratio $c_{2} / c_{1}$ remains close to unity for a significant portion of the slope. The examples below show progressively stronger coupling since the distance above the topography over which the speed ratio lies near to unity increases from each case to the next.

\subsection{A: Weak resonant coupling (initial depression wave)}

In case $A$ the middle layer thickness $\left(h_{2}\right)$ is thin and the lower layer $\left(h_{3}\right)$ is thicker than half of the middle layer $\left(h_{2} / 2\right)$ in the whole slope-shelf region. The incident mode- 1 wave is thus a depression solitary wave. Figure 2 shows the coefficients of the coupled$\mathrm{KdV}(2.23,2.24)$ model, with panels $(a, b)$ corresponding to the setup $h_{\text {gentle }}$ and $h_{\text {steep }}$ (panels $(a, d)$ in figure 1 ), respectively. The ratio $c_{2} / c_{1}$ is consistently small (covering the range from 0.42 (ocean edge) to 0.48 (shelf edge)). All the parameters are in the same range except for $h_{T}$, which is 4 times larger in the steep slope setup so the forced terms in $(2.23,2.24)$ are proportionally strengthened as in Liu et al. (2019a).

Figure 3 and 4 show the wave evolution under the gentle and steep slope setups respectively, using the coupled-KdV system for both mode- 1 and mode- 2 and the $\mathrm{KdV}$ model for mode- 1 alone. When the incident mode- 1 wave encounters the slope, a mode2 wave is generated propagating with a speed approximately $c_{2}$ that is much smaller 

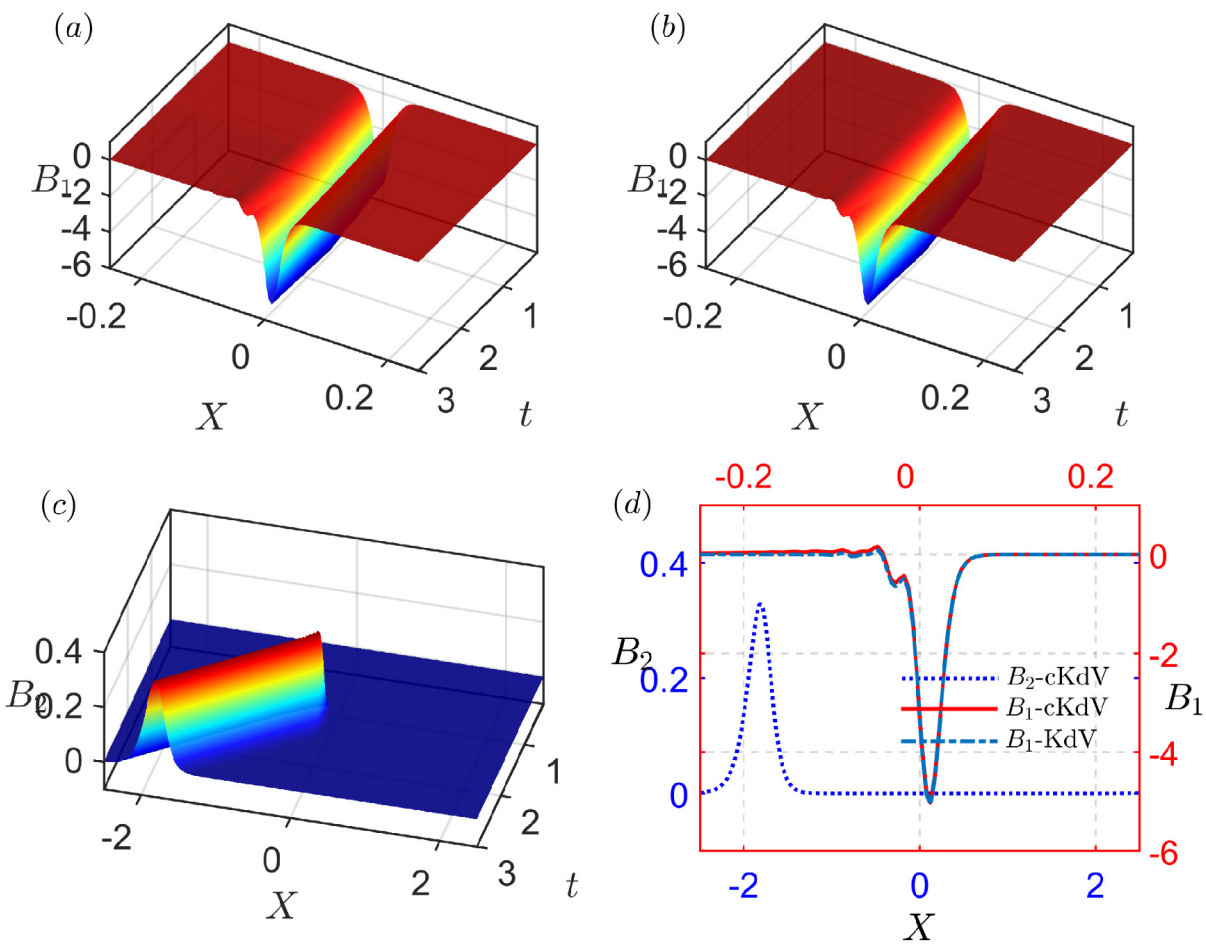

Figure 3. Case $A$. Wave evolution under the $\mathrm{KdV}(2.1)$ and coupled-KdV $(2.23,2.24)$ model for gentle slope gradient with an initial depression mode-1 solitary wave. Panels $(a, c)$ : mode-1 and mode- 2 wave evolution under the coupled-KdV system; panel $(b)$ : mode- 1 wave evolution under KdV equation; panel $(d)$ : the final state under simulation for these waves.

than the mode-1 linear wave speed $c_{1}$, and so in the mode- 1 coordinate system this generated mode- 2 wave travels to the left. Panel $(d)$ in figure 3 and 4 show the final state in this simulation where both the waves arrive at the shallow water region. For the mode- 1 wave, wave fission is starting to occur. There is no significant distinction between the two different topographic gradients. The feedback of the generated mode- 2 wave on the incident mode- 1 wave is too small to be seen. The generated mode- 2 waves are convex, with an amplitude $0.3 \mathrm{~m}$ in the gentle case and $1.2 \mathrm{~m}$ in the steep case, directly proportional to the change in the slope gradient.

\subsection{B: Moderate resonant coupling (polarity change for mode-1 wave)}

In case $B$ (see panels $(b, e)$ in the figure 1 ), the middle layer thickness is larger, and the upper and lower pycnoclines are much closer to the surface and bottom. The lower layer $\left(h_{3}\right)$ before the slope is thicker than a half of the middle layer $\left(h_{2} / 2\right)$, but thinner after the slope, and so a polarity change occurs for the incident mode- 1 wave that is initially a depression wave. Figure 5 show the parameters and coefficients of the coupled-KdV system. For this thicker middle layer the range of the speed ratio $c_{2} / c_{1}$ is from 0.58 (ocean) to 0.72 (shelf). The nonlinear coefficient $\nu_{1}$ of the mode- 1 wave passes through the critical value 0 at $x=0$ on the slope, and $\nu_{2}$ is now negative in contrast to case $A$. The relative mode- 2 wave speed $\Delta$ is smaller, so the distance between the incident mode- 1 wave and the generated mode- 2 wave is less over the same time interval. The 

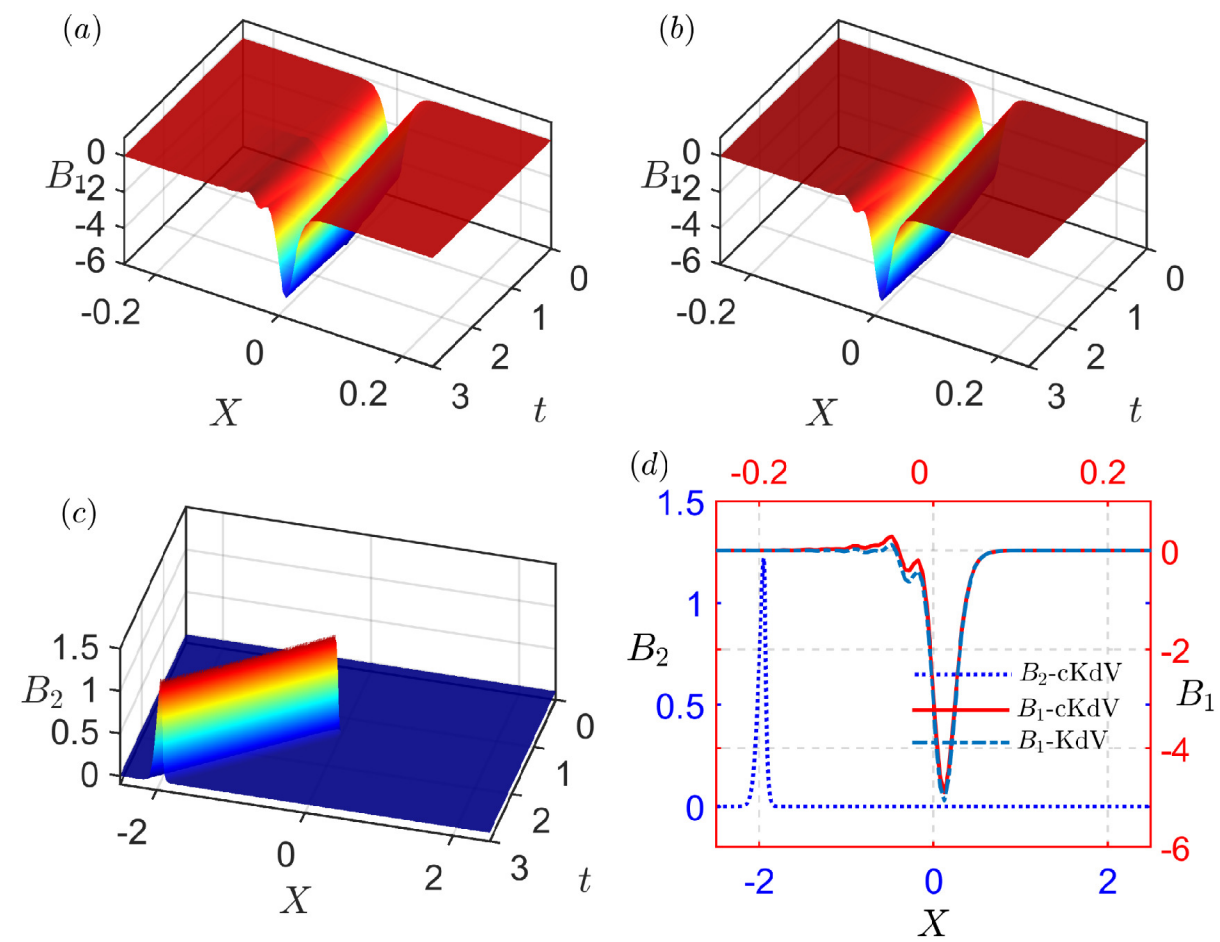

Figure 4. Case $A$. As in figure 3 except that the topographic slope is steeper.

coupling parameter $\gamma$ increases around 10 times while $\frac{\partial h}{\partial T}$ remains almost the same as in case $A$, and so coupling is considerably enhanced in this case $B$.

Figures 6 and 7 show the wave evolution for the gentle and steep slope setups (panels $(b, e)$ in figure 1$)$, respectively. The incident mode-1 changes polarity on the slope region, and in the $\mathrm{KdV}$ model we see an emerging rarefaction wave in the front followed by an undular bore (see panels $(b, d)$ in figure 6 and 7 ). However, in the coupled-KdV system, the mode- 1 wave evolution is now significantly affected by the generated mode- 2 wave. It changes polarity much faster with an elevation wave in the front and a depression wave at the back. The generated mode- 2 wave is now a concave shape, because of the opposite sigh of $\nu_{2}$ compared to case $A$. When the topographic gradient increases, the amplitude of the generated mode- 2 wave increases, which leads to a greater influence on the mode- 1 wave. We see that the elevated (depressed) part in figure 7 is much smaller (larger) than in figure 6 . Here, the mode- 2 wave propagates faster, so it takes longer to reach the same distance from the mode- 1 wave compared to case $A$.

\subsection{C: Strong resonant coupling (initial elevation wave)}

In case $C$ (panels $(c, f)$ in figure 1 ), the middle layer thickness increases further, so the upper and lower pycnoclines are very close to the surface and bottom, with the lower layer thickness $\left(h_{3}\right)$ thinner than a half of the middle layer $\left(h_{2} / 2\right)$ all through. The incident mode- 1 solitary wave is now an elevation wave. Figure 8 shows the parameters and coefficients of the coupled-KdV equations $(2.23,2.24)$. The mode- 2 and mode- 1 linear phase speeds are very close after the slope, the shelf edge value of the ratio is greater than 0.9 and so $c_{2} \approx c_{1}$. The nonlinear coefficients $\nu_{1}$ and $\nu_{2}$ stay positive and 
$(a)$
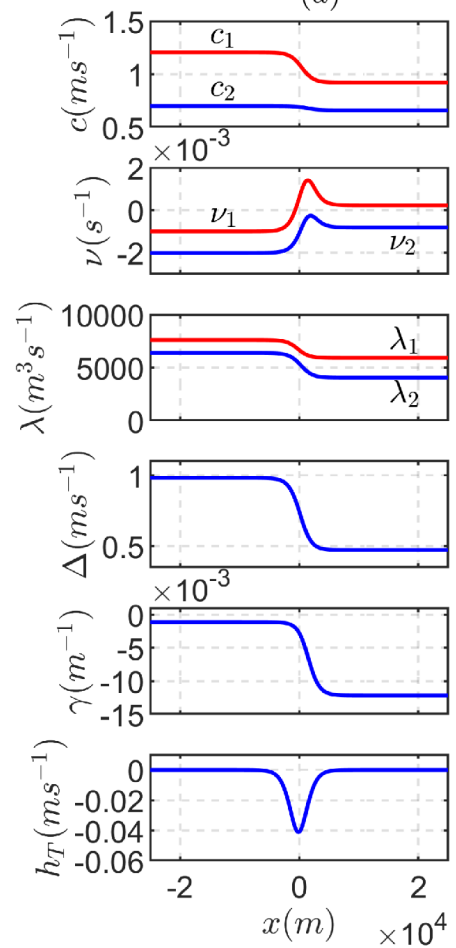

(b)
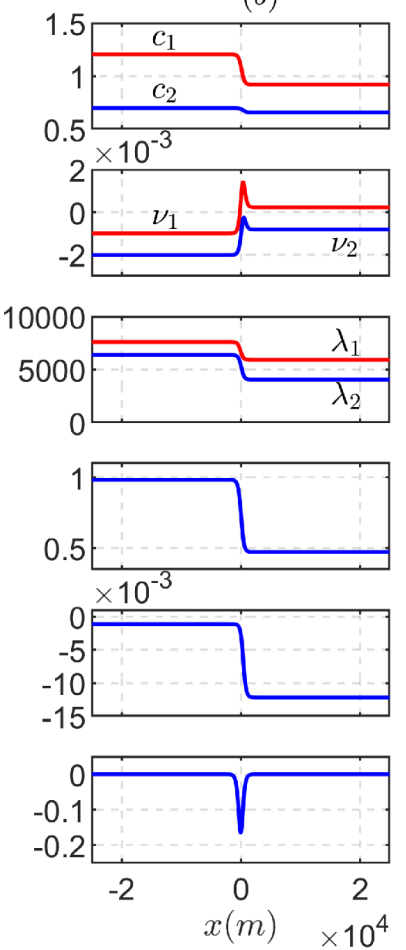

Figure 5. Case B. Coefficients of coupled-KdV $(2.23,2.24)$ model. Panels $(a, b)$ correspond to the gentle and steep slope respectively.

negative respectively, indicating an elevation mode- 1 wave and a concave mode- 2 wave. The relative speed $\Delta$ of the generated mode- 2 wave is approximately 0 after the slope, so the gap between mode- 1 and mode- 2 remains almost unchanged after crossing the slope region. $\gamma$ grows significantly by 100 times compared to case $A$, while $\frac{\partial h}{\partial T}$ does not change significantly, and so the coupling term is much stronger giving strong resonant coupling.

Figure 9 and 10 show the corresponding wave evolution under this gentle (3.1) and steep (3.2) setups with now a very thick middle layer. The incident elevation mode-1 wave fissions in the $\mathrm{KdV}$ model (see panels $(b, d)$ in figure 9 and 10) and the number of the generated solitary waves in the steep slope case are fewer than in the gentle slope case. For resonant coupling, amplitudes of the generated mode- 2 wave are much larger, comparable to the incident mode-1 wave or even larger (see panels $(c, d)$ in figure 9 and 10 ), while the mode- 1 wave evolution is suppressed by the generated mode- 2 wave with a much smaller leading wave followed by a downward wave tail (see panels $(a, d)$ ).

\section{The general qualitative behaviour}

The three cases of $\S 3$ establish the range of behaviours that occur when resonant interactions become significant. To assess the prevalence of these behaviours further simulations over a wider range of topographic slopes and layer thicknesses were performed. These simulations can be placed into one of the three qualitative classes: "depression", "polarity change" and "elevation", based on the closeness of the mode-1 evolution to 

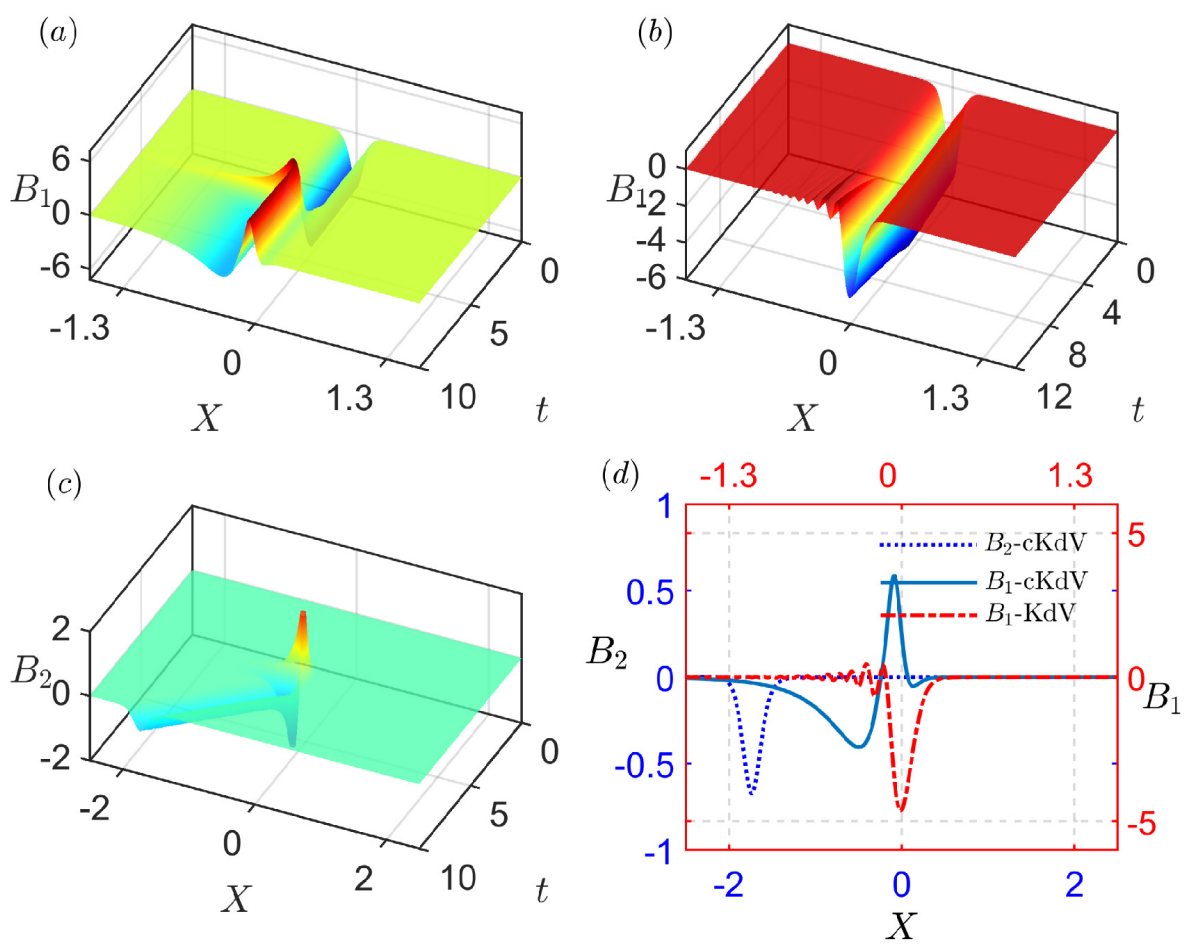

Figure 6 . Case $B$. As in figure 3 except that the layer thicknesses are different.

that in cases $A, B$ and $C$, shown in figures 11 and 12 as blue for "depression", red for "polarity change", and green for "elevation".

In figure 11 the topographic slope varies but the background stratification profiles (layer thicknesses) are the same as in cases $A, B$ and $C$. The flow evolution is the same as in the respective cases and the behaviour agree with those from the three detailed cases: with the layer thicknesses fixed the mode- 2 wave amplitude grows when the slope-width decreases, i.e. the gradient increases, and the rate of growth increases with decreasing slope-width. The maximum mode- 2 wave amplitude is inversely proportional to the slope-width entering through the topographic slope $\frac{\partial h}{\partial T}$ in the coupling term. For fixed slope width, the mode- 2 wave amplitude changes significantly due to the changes in stratification as in $\S 3$. The evolutions of $\S 3$ show also that the amplitude of the transmitted mode- 1 waves is relatively insensitive to the width of the slope region.

Figure 12 shows the connection between mode- 2 wave amplitude and stratification. The slope is either gentle (3.1) or steep (3.2) and only the layer thicknesses are altered. For the layer thicknesses in on-shelf region, since $h_{3}$ is marginally greater than $h_{1}$ (by $2 \mathrm{~m}$.), the evolution of the incident mode- 1 wave depends on the value of the ratio $h_{2} / h_{3}$. If $h_{2} / h_{3}<1$ (blue in figure 12) then the lower layer is uniformly thicker than the middle layer and so the mode- 1 wave remains a wave of depression, as in case $A$. If $1<h_{2} / h_{3}<3.25$ (red in figure 12) then the lower layer is thicker than the middle layer off-shelf but thinner on-shelf and so the mode- 1 polarity changes, as in case $B$. $h_{2} / h_{3}>3.25$ then the lower layer is uniformly thinner than the middle layer and mode-1 wave remains a wave of elevation, as in Case $C$. The mode- 2 wave generation and mode coupling in any given simulation depends on the cumulative effect on the ratio $c_{2} / c_{1}$ of the 

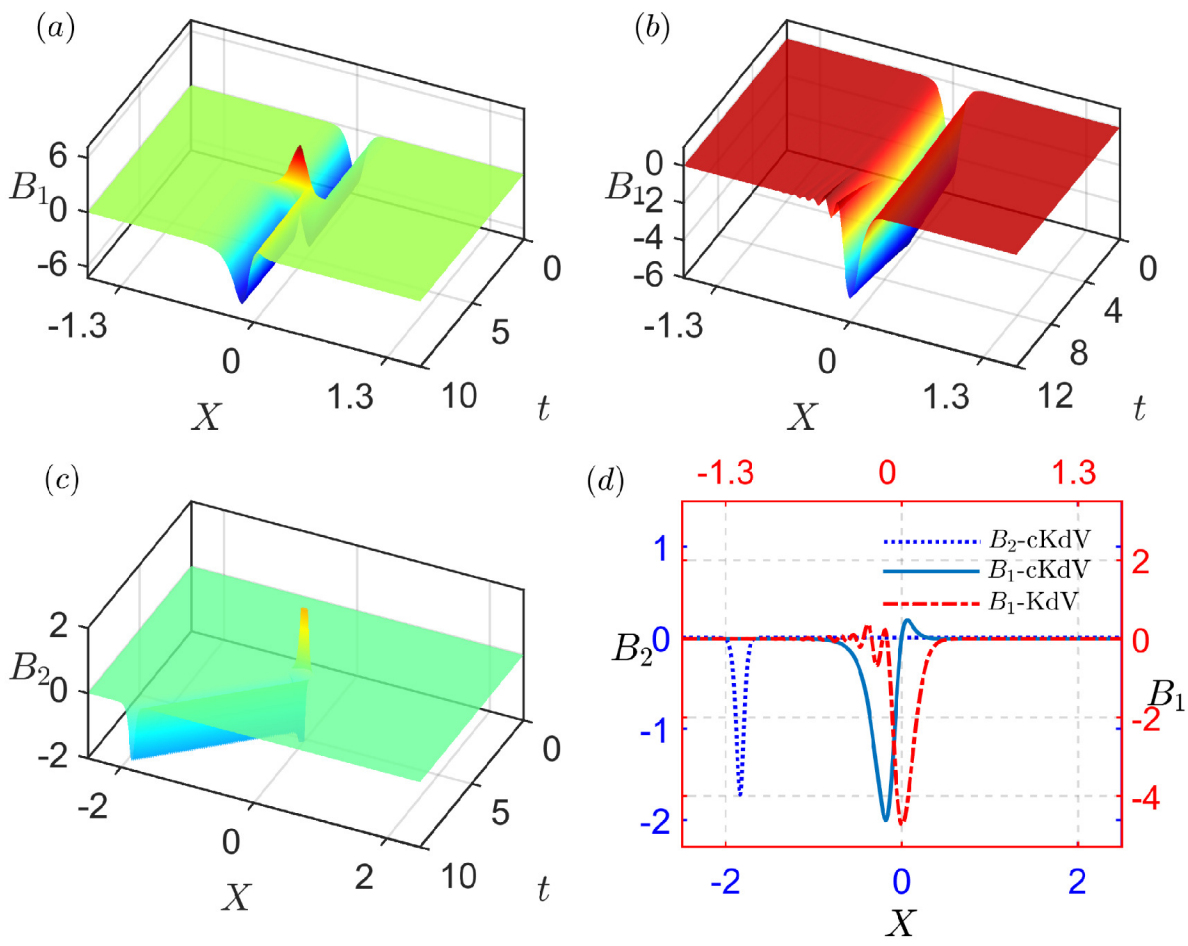

Figure 7 . Case $B$. As in figure 6 except that the topographic slope is steeper.

whole range of layer thicknesses over the slope, but for definiteness the results are plotted as a function of the ratio $h_{2} / h_{1} \approx h_{2} / h_{3}$, the layer thicknesses in the on-shelf region. The maximum mode- 2 wave amplitude increases as the layer thickness ratios $h_{2} / h_{1}$ and $h_{2} / h_{3}$ grow. As the middle layer thickness increases the behaviour moves from that of case $A$ to case $B$ and then case $C$. The growth rate increases rapidly with increasing $h_{2} / h_{1}$ for case $A$ and $B$ behaviour as both the lower and upper limits of the range of $c_{2} / c_{1}$ increase. For case $C$ flows the lower limit decreases as $h_{2} / h_{1}$ increases, while the upper limit increases. The combined effect of $\gamma$, speed ratio $c_{2} / c_{1}$ and slope gradient $\frac{\partial h}{\partial T}$ means that the forcing, although stronger than in case $A$ and $B$ flows, does not increase with $h_{2} / h_{1}$. More importantly, for these flows the relative speed $\Delta$, which decreases as $h_{2} / h_{1}$ increases, is small. Linear generation, as described in $\S 2.3$, would continue the trend of the case $A$ and $B$ flows and the generated wave would be arbitrarily large. In the coupled $\mathrm{KdV}$ system nonlinearity limits the size of this near-resonant response. As noted in the discussion of case $C$, dispersive effects then lead to fission of the generated larger-amplitude mode- 2 wave. The amplitude plotted in figure 12 is that of the leading mode-2 after fission and so does not capture all the incident energy transferred into mode-2 disturbances. This can be seen in the neighbourhood of $h_{2} / h_{1} \approx 4$ in figure 12 . More energy is transferred to mode- 2 than for smaller values of $h_{2} / h_{1}$ but nonlinearity and dispersion split the disturbance into separate waves with the leading wave having smaller amplitude than the single wave generated for smaller $h_{2} / h_{1}$.

The largest amplitude generated mode- 2 waves from resonant interactions are concave waves generated by incident elevation mode- 1 waves: the combination of a near-surface and near-bottom pycnocline gives both a large range for the ratio $c_{2} / c_{1}$ over the slope 
$(a)$
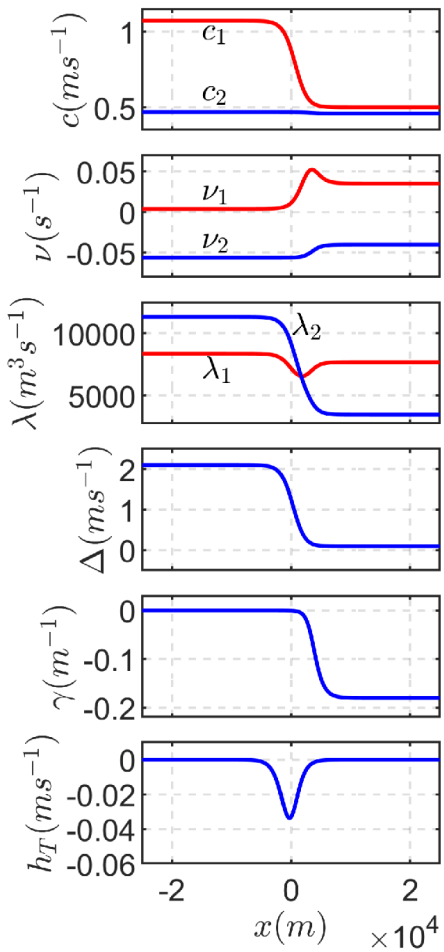

(b)
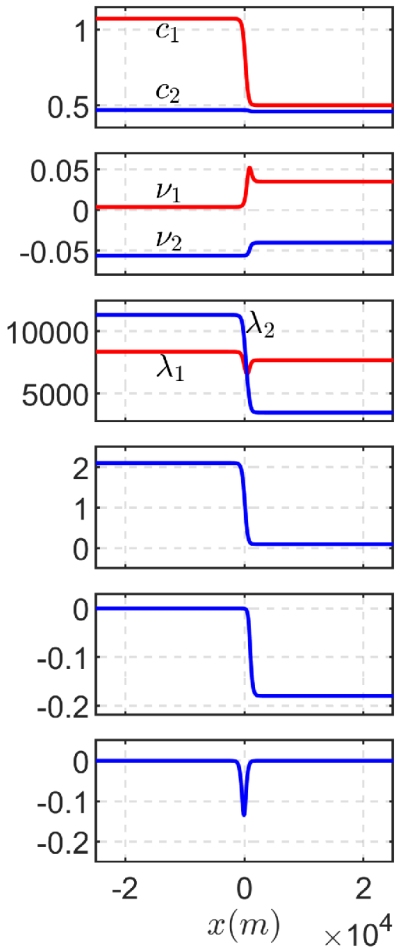

Figure 8. Case $C$. Coefficients of coupled-KdV $(2.23,2.24)$ model. Panels $(a, b)$ are corresponding to the gentle and steep slope in $C$ setup, respectively.

and values of $c_{2} / c_{1}$ near unity. In agreement with figure 11, for a given value of the ratio $h_{2} / h_{1}$ the mode- 2 amplitude over a steep slope is greater than that over a gentler slope.

\section{Summary and discussion}

In this paper we have extended the study by Liu et al. (2019a) for the generation of mode- 2 internal waves when a mode- 1 internal solitary wave encounters variable topography to the case when there is resonant coupling of the two modes, defined as when the speed ratio $c_{2} \approx c_{1}$. In this resonant regime the linearised theory of Liu et al. (2019a), in which the mode- 1 wave forces a mode- 2 wave, is replaced by coupled $\mathrm{KdV}$ equations (2.23.2.24) which allow for feedback from the generated mode- 2 wave to the incident mode- 1 wave. Three representative cases $(A, B, C)$ have been examined in detail using a three-layer fluid system with different layer thicknesses with each case containing two subcases of gentle and steep topographic slopes. Subsequent simulations could then be classified according to their closeness to these three typical cases. Table 2.4 and figure 1 show the profiles of the three-layer setup; total depth before and after the slope are fixed; the layer thickness $\left(h_{1}, h_{2}, h_{3}\right)$ are varied, so that the pycnoclines become closer to the top and bottom, which leads to $c_{2} \approx c_{1}$ (Liu et al. (2019b)).

The response is determined by the range over the topography of the ratio $c_{2} / c_{1}$ which is form 0.42 at the ocean edge of the slope to 0.48 at the shelf edge for case A, from 0.58 (ocean) to 0.72 (shelf) for case B and from 0.44 (ocean) to 0.92 (shelf) for case C. The coupling parameter $\gamma$ in the coupled KdV system consequently increases around tenfold 


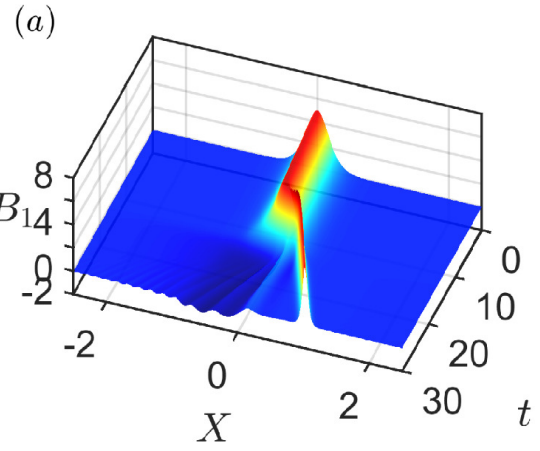

$(c)$

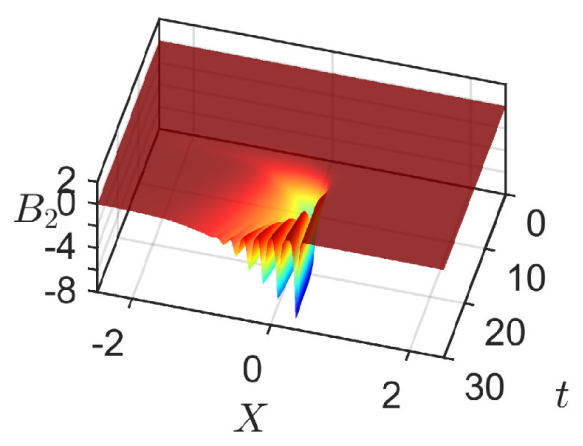

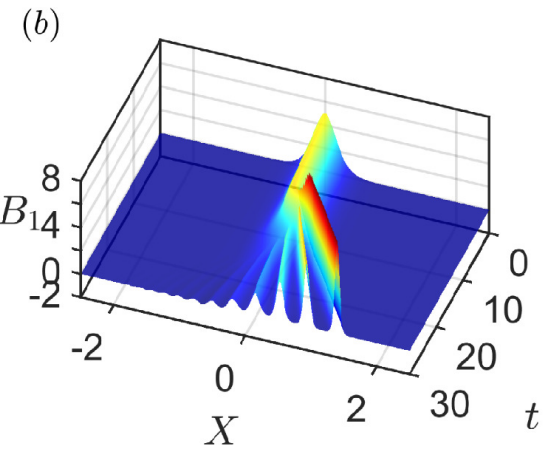

$(d)$

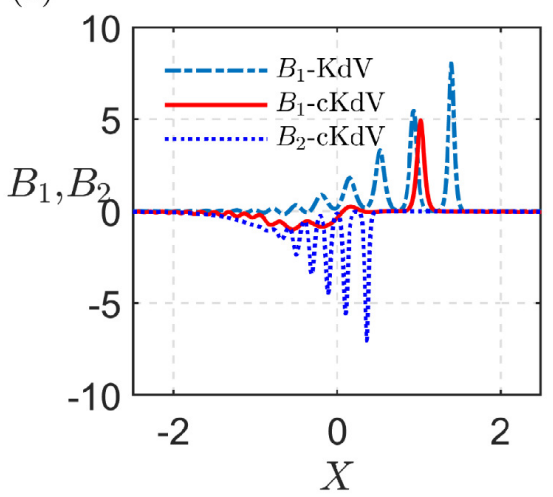

Figure 9. Case $C$. As in figure 3 except that the layer thicknesses are different.

from case A to case $\mathrm{B}$ and then again to case $\mathrm{C}$, while the speed $\Delta$ of mode- 2 relative to mode- 1 approaches 0 as the speed ratio $c_{2} / c_{1}$ increases. In all cases we set the amplitude of the incident mode- 1 wave to be $5 \mathrm{~m}$. In cases $A$ and $B$, initially it is a depression wave, while in case $B$, polarity change happens when mode- 1 wave encounters the topographic slope, and in case $C$, initially it is an elevation wave. This can be seen from the variation of the nonlinear coefficients in the coupled $\mathrm{KdV}$ equations. (i): In figure 2 (case $A$ ), for mode- 1 wave, $\nu_{1}$ is negative throughout, and nonlinear coefficient of mode- 2 wave $\nu_{2}$ has the opposite sign. Hence we have a depression mode- 1 wave with a convex mode- 2 wave. (ii): In case $B$ (figure 5 ), $\nu_{2}$ is negative, but $\nu_{1}$ changes sign from negative to positive, indicating that polarity conversion occurs for the mode- 1 wave, and the mode- 2 wave is concave, although a convex mode- 2 wave appears momentarily when mode- 1 wave first comes up against the slope. (iii): The last case is quite different to case $A, \nu_{1}$ positive and $\nu_{2}$ negative, so the initial incident mode- 1 wave is an elevation wave and then generates a concave mode- 2 wave after interaction with the continental slope.

The magnitudes of the amplitudes of the generated mode- 2 waves increases (from $0.3 /, m$ to $10 /, m$ ) when the range of the ratio $c_{2} / c_{1}$ and/or the topographic slope grow, which is consistent with Liu et al. (2019a). The mode-2 wave amplitude is proportional to the slope gradient $\left(\frac{\partial h}{\partial T}\right)$ with a fixed range of speed ratio (the layer thicknesses are fixed). As the generated mode- 2 wave amplitude becomes larger, its effect on the incident mode- 1 wave is enhanced, either promoting or suppressing the evolution of mode- 1 wave, see cases $B$ and $C$. This can be clearly seen when comparing the resonant coupling mode- 
(a)

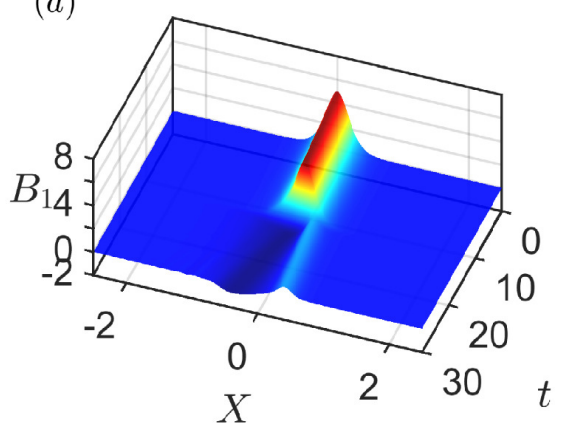

(c)

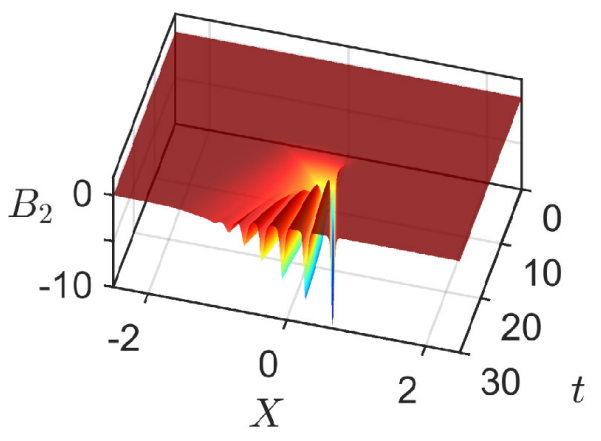

$(b)$

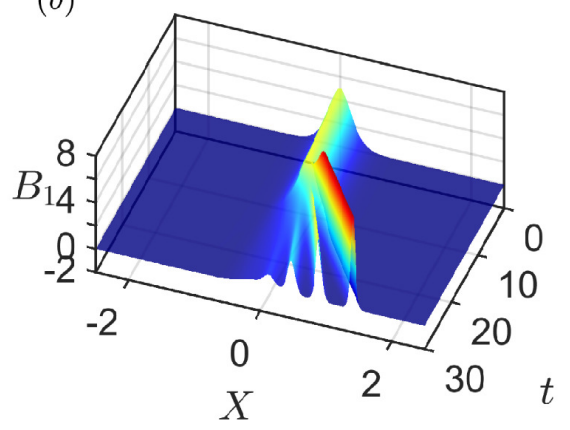

$(d)$

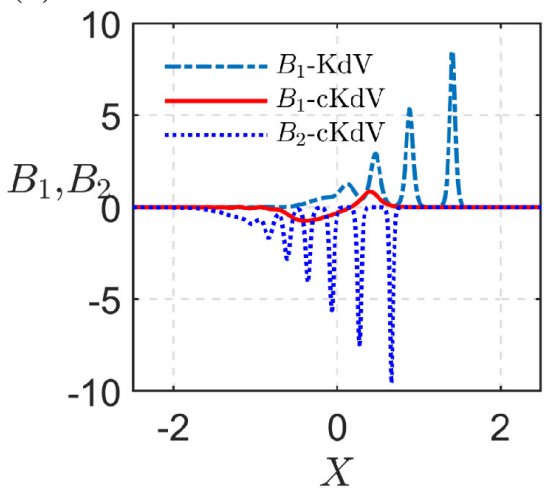

Figure 10. Case $C$. As in figure 9 except that the topographic slope is steeper.

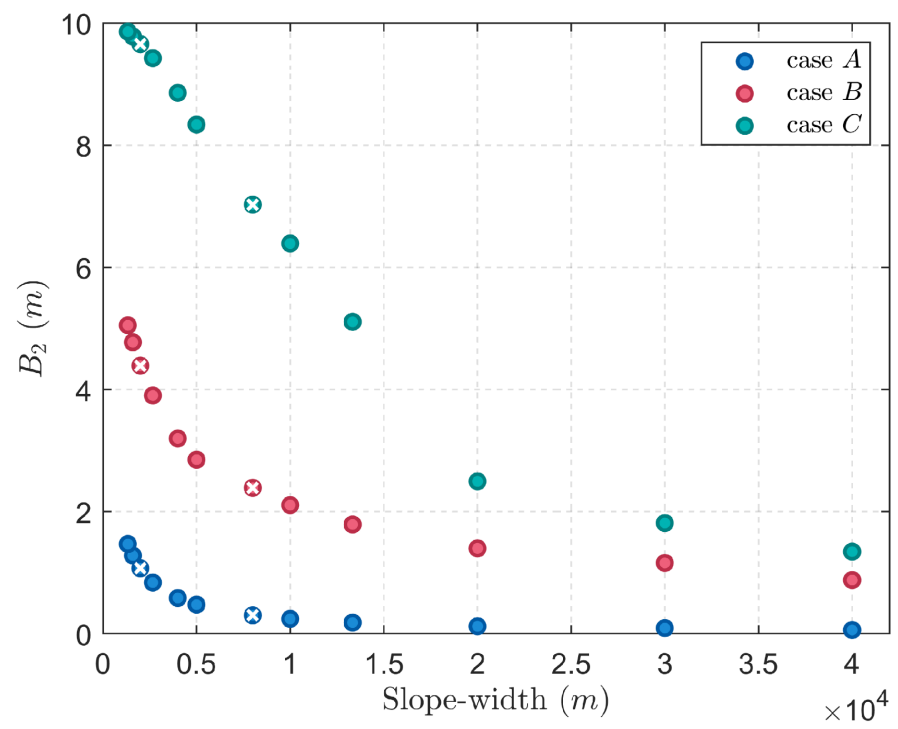

Figure 11. The maximum mode- 2 amplitude $\left(B_{2}\right)$ for different topographic gradients for the stratification (layer thicknesses) of cases $A, B$ and $C$. The points with white crosses represent the cases with the same slope width and stratification in $\S 3$. 


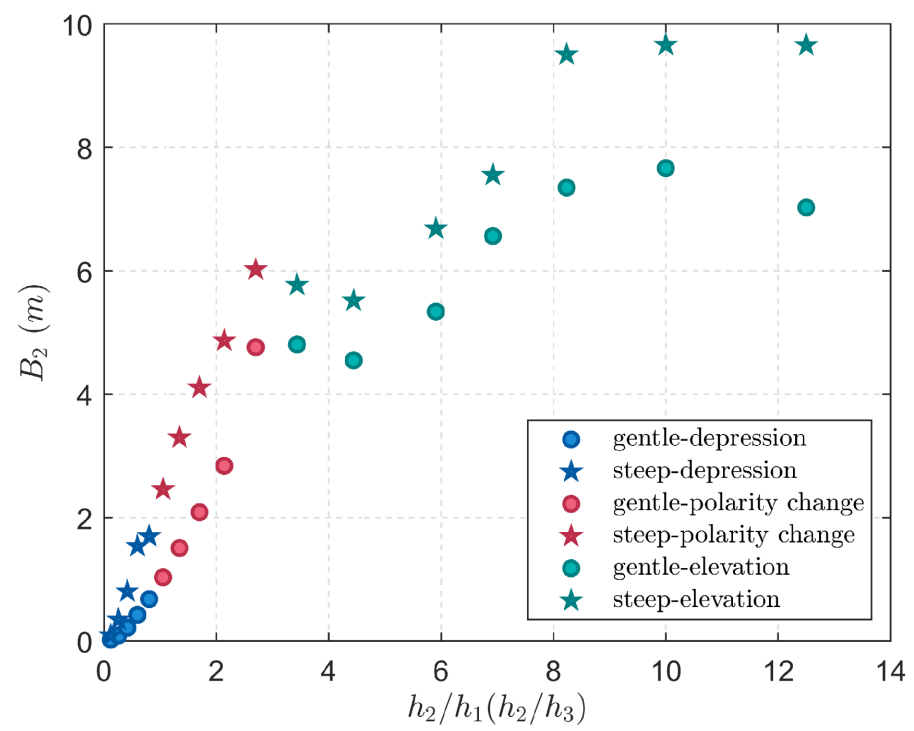

Figure 12. The maximum mode-2 amplitude $\left(B_{2}\right)$ for different stratifications for gentle (3.1) and steep (3.2) slopes. The top layer thickness $\left(h_{1}\right)$ is close to that of the bottom $\left(h_{3}\right)$ on the shelf so the ratios $\left(h_{2} / h_{1}, h_{2} / h_{3}\right)$ can be treated as equal. The descriptions "depression", "polarity change" and "elevation" refer to the evolution of the incident mode-1 wave.

1 wave profile with the wave shape under $\mathrm{KdV}$ model where there is no coupling, see panels $(b, d)$ in figures $3,4,6,7,9$ and 10 . Also when $c_{2} \approx c_{1}$, the relative speed $\Delta \approx 0$, so the distance between the generated mode- 2 wave and the incident mode- 1 wave is very close, enhancing the feedback.

The discussion in $\S 4$ of the maximum mode- 2 wave amplitude for a wide range of slopes and stratifications reveals the dependence on topographic slope $\frac{\partial h}{\partial T}$ and the parameter $\gamma$ in addition to the importance of the range of values over the slope of the ratio $c_{2} / c_{1}$. The most conducive conditions for resonant mode- 2 wave generation are a near-surface and near-bottom pycnocline and steep topography, giving concave mode- 2 waves. To date most observed mode- 2 waves are convex as the background stratifications for the observations tend to be those on which mode- 1 waves of depression are more common than those of elevation. Further most oceanic pycnoclines are thin and so are more likely to support a convex rather than a concave mode- 2 wave. Another possibility, beyond the current analysis, is that the larger concave mode- 2 waves are unstable and thus difficult to observe. The mode- 2 convex waves in the present analysis that appear when $h_{2} / h_{1} \approx h_{2} / h_{3}$ is small are of negligible amplitude due to the middle layer being too thin to be treated as fully three-layer fluid and so not providing the background for a mode-2 wave.

The circumstances when resonant interactions occur are not commonly observed, and in most cases the non-resonant theory of Liu et al. (2019a) applies and only smallamplitude mode- 2 waves will be found. The results here show nevertheless that it is not necessary to be precisely at resonance to generate significant mode- 2 wave amplitudes and feedback onto mode-1. Although the results here are for a three-layer model, the key parameter is the range over the slope of the speed ratio $c_{2} / c_{1}$ which can be readily found for any density stratification. Mode- 2 wave amplitudes are larger when the topographic 
slope is steeper, indicating that a combination of near-resonance and steep topographic slope should lead to significant mode-2 generation.

The general results indicate that resonant coupling of two modes by topography tends to generate large concave mode- 2 waves from either an incident elevation mode- 1 wave or a depression mode-1 wave through polarity change, and convex waves with considerable amplitude compared to an incident depression mode- 1 wave. However many other unrelated mechanisms, like tidal forcing and frontal intrusions, are candidates for generating these waves and, in particular for convex mode-2, will certainly be more relevant in some locations. Yang et al. (2009) summarized five potential generation mechanisms for mode-2 waves; three of which involved mode- 1 waves through breaking instability, reflection, and interaction with topography (a sill), while two were a gravity current intrusion and flow over negative topography. Their observations of mode-2 internal solitary waves on the continental slope of the northern South China Sea also indicated that mode- 2 waves showed notable seasonal variations: in summer, $90 \%$ of observed mode- 2 waves appeared after mode- 1 waves, but only $28 \%$ in winter. They noted that mode- 2 waves in summer may be related to the shoaling process of a mode- 1 internal solitary wave or internal tide (an internal wave with tidal frequency), while mode- 2 wave generation in winter could be due to density variations. Even at the same location the generation mechanism for mode- 2 waves can have seasonal differences.

\section{Declaration of interests}

The authors report no conflict of interest.

\section{REFERENCES}

Akylas, T. R. and Grimshaw, R. H. J. (1992). Solitary internal waves with oscillatory tails. Journal of Fluid Mechanics, 242:279-298.

Carr, M., Stastna, M., Davies, P. A., and van de Wal, K. J. (2019). Shoaling mode-2 internal solitary-like waves. Journal of Fluid Mechanics, 879:604-632.

Cheng, M.-H., Hsieh, C.-M., Hsu, J. R.-C., and Hwang, R. R. (2017). Transformation of mode-2 internal solitary wave over a pseudo slope-shelf. AIP Advances, 7(9):095309.

Deepwell, D. and Stastna, M. (2016). Mass transport by mode-2 internal solitary-like waves. Physics of Fluids, 28(5):056606.

Deepwell, D., Stastna, M., Carr, M., and Davies, P. A. (2017). Interaction of a mode-2 internal solitary wave with narrow isolated topography. Physics of Fluids, 29(7):076601.

Deepwell, D., Stastna, M., Carr, M., and Davies, P. A. (2019). Wave generation through the interaction of a mode- 2 internal solitary wave and a broad, isolated ridge. Phys. Rev. Fluids, 4:094802.

Farmer, D. M. and Smith, J. D. (1980). Tidal interaction of stratified flow with a sill in Knight Inlet. Deep Sea Research Part A. Oceanographic Research Papers, 27(3):239-254.

Gear, J. A. and Grimshaw, R. (1984). Weak and strong interactions between internal solitary waves. Studies in Applied Mathematics, 70(3):235-258.

Griffiths, S. D. and Grimshaw, R. H. J. (2007). Internal tide generation at the continental shelf modeled using a modal decomposition: two-dimensional results. J. Phys. Ocean., pages 428-451.

Grimshaw, R. (1981). Evolution equations for long nonlinear internal waves in stratified shear flows. Stud. Appl. Math, 65:159-188.

Grimshaw, R. (2007). Internal solitary waves in a variable medium. Gesellschaft fur Angewandte Mathematik, 30:96-109.

Grimshaw, R., Pelinovsky, E., Talipova, T., and Kurkina, A. (2010). Internal solitary waves: propagation, deformation and disintegration. Nonlinear Processes in Geophysics, 17:633649. 
Helfrich, K. R. and Melville, W. K. (1986). On long nonlinear internal waves over slope-shelf topography. Journal of Fluid Mechanics, 167:285-308.

Helfrich, K. R. and Melville, W. K. (2006). Long nonlinear internal waves. Ann. Rev. Fluid Mechanics, 38:395-425.

Klymak, J. M. and Moum, J. N. (2003). Internal solitary waves of elevation advancing on a shoaling shelf. Geophysical Research Letters, 30(20).

Lamb, K. G. and Warn-Varnas, A. (2015). Two-dimensional numerical simulations of shoaling internal solitary waves at the ASIAEX site in the South China Sea. Nonlinear Processes in Geophysics, 22(3):289-312.

Liu, A. K., Chang, Y. S., Hsu, M.-K., and Liang, N. K. (1998). Evolution of nonlinear internal waves in the East and South China Seas. Journal of Geophysical Research: Oceans, 103(C4):7995-8008.

Liu, A. K., Su, F.-C., Hsu, M.-K., Kuo, N.-J., and Ho, C.-R. (2013). Generation and evolution of mode-two internal waves in the South China Sea. Continental Shelf Research, 59:18-27.

Liu, Z., Grimshaw, R., and Johnson, E. (2019a). Generation of mode 2 internal waves by interaction of mode 1 waves with topography. J. Fluid Mech., 880:799-830.

Liu, Z., Grimshaw, R., and Johnson, E. (2019b). The interaction of a mode-1 internal solitary wave with a step and the generation of mode-2 waves. Geophys. Astro. Fluid. Dyn.

Maderich, V., Jung, K. T., Terletska, K., and Kim, K. O. (2017). Head-on collision of internal waves with trapped cores. Nonlinear Processes in Geophysics, 24(4):751-762.

Magalhaes, J. M. and Da Silva, J. C. B. (2018). Internal solitary waves in the Andaman Sea: New insights from SAR imagery. Remote Sensing, 10(6).

Maxworthy, T. (1980). On the formation of nonlinear internal waves from the gravitational collapse of mixed regions in two and three dimensions. Journal of Fluid Mechanics, 96(1):47-64.

Mehta, A. P., Sutherland, B. R., and Kyba, P. J. (2002). Interfacial gravity currents . II . Wave excitation. Phys. Fluids.

Moum, J. N. and Smyth, W. D. (2006). The pressure disturbance of a nonlinear internal wave train. Journal of Fluid Mechanics, 558:153-177.

Ramp, S. R., Yang, Y. J., Reeder, D. B., and Bahr, F. L. (2012). Observations of a mode-2 nonlinear internal wave on the northern Heng-Chun Ridge south of Taiwan. Journal of Geophysical Research: Oceans, 117(C3).

Rayson, M. D., Jones, N. L., and Ivey, G. N. (2019). Observations of large-amplitude mode2 nonlinear internal waves on the Australian North West Shelf. Journal of Physical Oceanography, 49(1):309-328.

Shroyer, E. L., Moum, J. N., and Nash, J. D. (2010). Mode-2 waves on the continental shelf: Ephemeral components of the nonlinear internal wave field. J. Geophys. Res. Oceans, 115(C7):C0700.

Stastna, M., Olsthoorn, J., Baglaenko, A., and Coutino, A. (2015). Strong mode-mode interactions in internal solitary-like waves. Physics of Fluids, 27(4):046604.

Terletska, K., Jung, K. T., Talipova, T., Maderich, V., Brovchenko, I., and Grimshaw, R. (2016). Internal breather-like wave generation by the second mode solitary wave interaction with a step. Phys. Fluids, 28:116602.

Vanden-Broeck, J. and Turner, R. E. L. (1992). Long periodic internal waves. Physics of Fluids A: Fluid Dynamics, 4(9):1929-1935.

Vlasenko, V. I. and Hutter, K. (2001). Generation of second mode solitary waves by the interaction of a first mode soliton with a sill. Nonlin. Processes Geophys., 8:223-239.

Yang, Y. J., Fang, Y. C., Chang, M.-H., Ramp, S. R., Kao, C.-C., and Tang, T. Y. (2009). Observations of second baroclinic mode internal solitary waves on the continental slope of the northern South China Sea. J. Geophys. Res. Oceans, 114:C005318.

Yang, Y. J., Fang, Y. C., Tang, T. Y., and Ramp, S. R. (2010). Convex and concave types of second baroclinic mode internal solitary waves. Nonlinear Processes in Geophysics, 17(6):605-614.

Yuan, C., Grimshaw, R., and Johnson, E. (2018). The evolution of second mode internal solitary waves over variable topography. Journal of Fluid Mechanics, 836:238-259.

Zhao, Z. and Alford, M. H. (2006). Source and propagation of internal solitary waves Cambridge University Press 
in the northeastern South China Sea. Journal of Geophysical Research: Oceans, 111(C11):C11012. 